\title{
High-Field Saturation Behavior of Field-Grading Materials
}

\author{
G. Eriksson, H. Greijer, M. Pradhan \\ Corporate Research \\ $\mathrm{ABB} \mathrm{AB}$ \\ Västerås, Sweden \\ goran.z.eriksson@se.abb.com
}

\begin{abstract}
The nonlinear field-grading materials (FGM) exhibit an electric conductivity that increases with the applied electric field. They can be used to reduce excessive field stress in critical regions of high-voltage equipment. Computer modeling of the field-grading effect is usually performed employing simplified analytical expressions for the conductivity as function of the electric field strength $E$. In particular, the conductivity is allowed to increase with $E$ without limits. From measurements on actual FGMs, however, it is known that above a certain field strength the conductivity ceases to increase further. In this paper we study how the saturation of the conductivity affects the overall stress reduction and the heating of the material.
\end{abstract}

Keywords-field-grading material; nonlinear conductivity; saturation; electric field; heating

\section{INTRODUCTION}

In various high-voltage applications, such as rotating machines, cable terminations and cable joints, there frequently occur localized regions with very high electric field stress. This may under unfavorable conditions lead to electric flashover or breakdown, as well as thermal breakdown due to excessive resistive heating, especially in the vicinity of corners and sharp edges. One remedy is to distribute the voltage drop between conductors more evenly, thus resulting in a lower maximum field amplitude close to the critical point of interest. This applies to both DC as well as AC and transient conditions.

A commonly used method to provide the desired field grading is to insert a slightly conducting material between the conductors [1], [2]. The electrons will then redistribute so as to reduce the highest voltage gradients and in this way lower the peak electrical stress. For DC applications, it is essential that the field-grading material be in contact with both the high- and low-voltage conductors. For AC and transient field grading, however, it is strictly speaking only required to occupy the space where the field reduction is desired. In all cases currents will flow inside the FGM, resulting in resistive heating. Care must therefore be taken to avoid overheating. Thermal runaway, caused by a significant temperature dependence of the conductivity, may further increase this risk [1]. It follows from this that the FGM must have a conductivity high enough to provide the desired field grading but not so high that unacceptable heating will occur.
Although an ordinary linear material with a small, constant conductivity is capable of lowering the maximum field-stress values significantly, the field distribution will not become completely homogeneous. To provide even better field grading, composites having a field-dependent conductivity have been developed and are now being routinely used in industrial applications [2]. Nonlinear FGM materials of this kind usually consist of a base polymer, e.g. EPDM or SiR, in combination with a filler, such as $\mathrm{SiC}$ or $\mathrm{ZnO}$, providing the grain interfaces that give the desired nonlinear properties.

The characteristic feature of these materials is that the conductivity is constant at low field strengths but starts to increase rapidly above a certain threshold field. There are several ways of analytically expressing this dependence; one commonly used formula for the DC conductivity is

$$
\sigma_{D C}=\sigma_{0}\left(1+\left(\frac{E}{E_{c}}\right)^{\alpha}\right),
$$

where $\sigma_{0}$ is the conductivity at low fields, $E_{c}$ is the critical threshold field above which the conductivity increases rapidly, and the parameter $\alpha$ describes the rate of increase. The fieldgrading efficiency for each application can be optimized by tailoring the material composition in such a way that the resulting shape of the conductivity curve results in the smallest possible field stress. Because the exact level of stress reduction is determined by strongly nonlinear equations, it becomes very difficult to predict without employing numerical simulations. Also geometry has a big influence. Formulations such as (1) are, however, straightforward to use in many electric-field simulation tools.

The usual situation is that in order to avoid excessive heating the FGM is not driven unnecessarily far into its nonlinear regime. Hence, simple expressions like (1), where $\sigma_{D C}$ formally is allowed to increase without limits, can be used without difficulties. At very high stress levels, however, real materials cannot increase their conductivity above a certain level; the material is said to saturate. This effect has been observed experimentally, although the design of the measurement method becomes rigorous due to the risk of flashovers or breakdown at the high voltage levels required for achieving high-field amplitudes [3], [4]. The conductivity of FGMs increases with increased stress due to nonlinearity 
arising from particle-particle contacts in case of SiC-based FGM, and grain-boundary effects in ZnO-based FGM [2]. However, at very high fields the conductivity of FGM is governed by the bulk conductivity of the particles themselves ( $\mathrm{SiC}$ or $\mathrm{ZnO})$ and is referred to as saturated conductivity.

The aim of the present paper is to quantify how the saturation of conductivity may affect the field-grading efficiency in real-life applications. For this purpose we use a 2D model of a high-voltage bushing where much of the design has large similarities with high-voltage cable terminations. In order not to involve questions on how an FGM reacts to timedependent applied fields, we focus here on only the DC stress distribution [5].

\section{DC CONDUCTIVITY PARAMETRIZATION}

The DC conductivity described by (1) increases without limits as $E$ grows. One way, out of many, to include the effect of saturation is to use the modified expression

$$
\sigma_{D C}=\sigma_{0}\left(1+\left(\frac{E}{E_{c}}\right)^{\alpha}\right)\left(1+\left(\frac{E}{E_{s}}\right)^{\alpha}\right)^{-1}
$$

where $E_{s}>E_{c}$ is the field amplitude where saturation starts to set in. Note that $\sigma_{D C} \approx \sigma_{0}$ for $E<<E_{c}, \sigma_{D C} \approx \sigma_{0}\left(E / E_{c}\right)^{\alpha}$ for $E_{c}<<$ $E<<E_{s}$, and $\sigma_{D C} \approx \sigma_{0}\left(E_{s} / E_{c}\right)^{\alpha}$ for $E>>E_{s}$.

In the simulation examples that follow we use the typical values $\sigma_{0}=1 \times 10^{-14} \mathrm{~S} / \mathrm{m}$ and $\alpha=5$ for the low-field conductivity and nonlinearity coefficient, respectively [3], [4]. By varying $E_{c}$ and the ratio $E_{s} / E_{c}$ different conductivity curves are obtained, see Fig. 1.

\section{APPLICATION MODEL}

In order to study the saturation effect we choose a $2 \mathrm{D}$ axisymmetric model of a $1 \mathrm{~m}$ long, oil-cooled DC bushing, where the inner conductor of a shielded high-voltage cable is led through a conducting wall and into the oil-filled volume, see Fig. 2. The voltage difference between the inner conductor and the end of the grounded cable shield is evenly distributed along an annular vertical tube made of FGM, just as

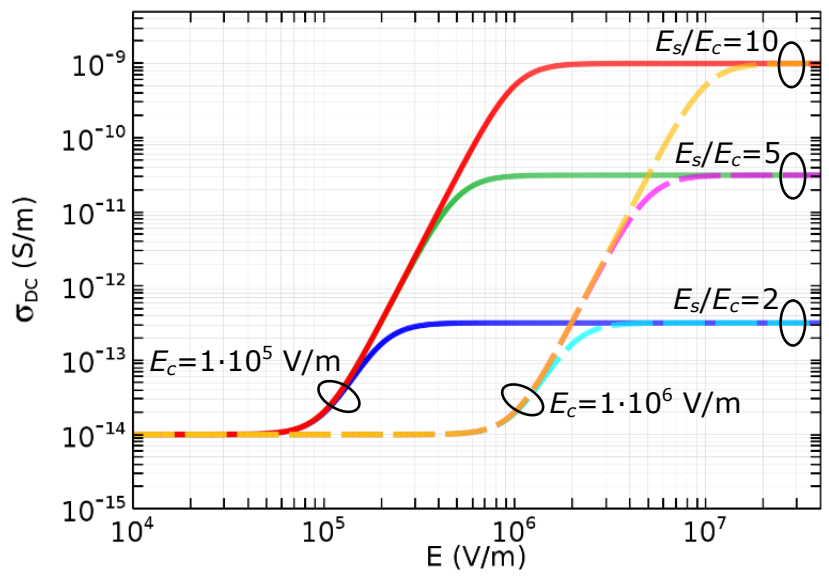

Fig. 1. Parametrization of the field dependent DC conductivity.
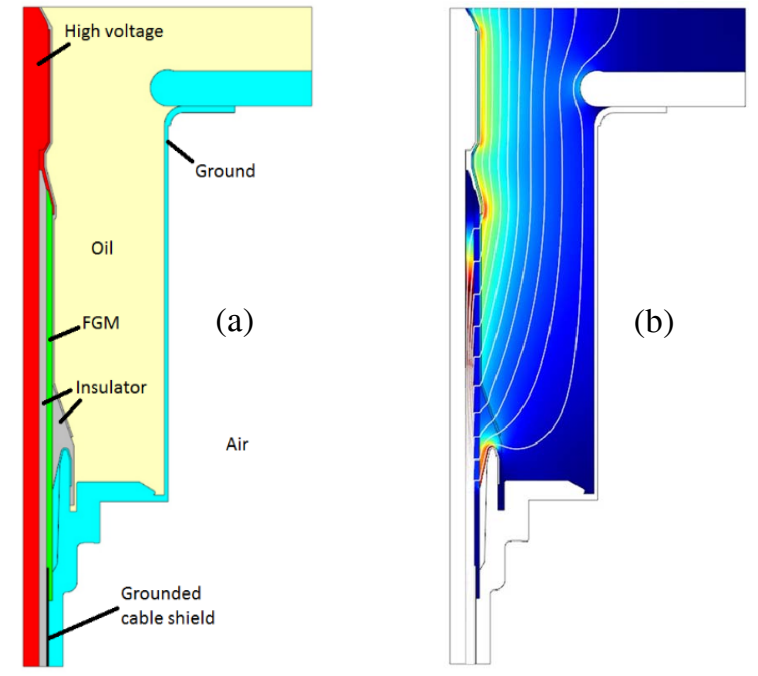

Fig. 2. An oil-filled high-voltage bushing (a), and the electric field amplitude and equipotential curve distribution (b) [6]. The FGM annular tube (shown green in (a)) distributes the equipotential curves evenly in the vertical $(z-)$ direction, thus reducing the maximum tangential electric stress.

is commonly seen in cable termination designs. This particular bushing model has been previously used to study the coupled electromagnetic-thermal problem and is described in some detail in [6]. For the purpose of the present work, we only consider the solution of the electrostatic field problem. The applied voltage in the simulations is $250 \mathrm{kV}$, a value chosen only because it enables us to illustrate the general effect of conductivity saturation on the field-grading efficiency. For the simulations the finite-element software COMSOL Multiphysics ${ }^{\mathrm{TM}}$ has been used [7].

\section{FGM WITHOUT SATURATION}

Let us first illustrate how a linear FGM, having a constant conductivity, can be used to reduce the maximum field stress. In Fig. 3 we plot the tangential electric field $E_{\text {tan }}$ and the local resistive heating density $Q_{\text {res }}$ along the outer surface of the FGM tube. This is done both for the case when there is no FGM tube present and for the case with a tube made of a linear

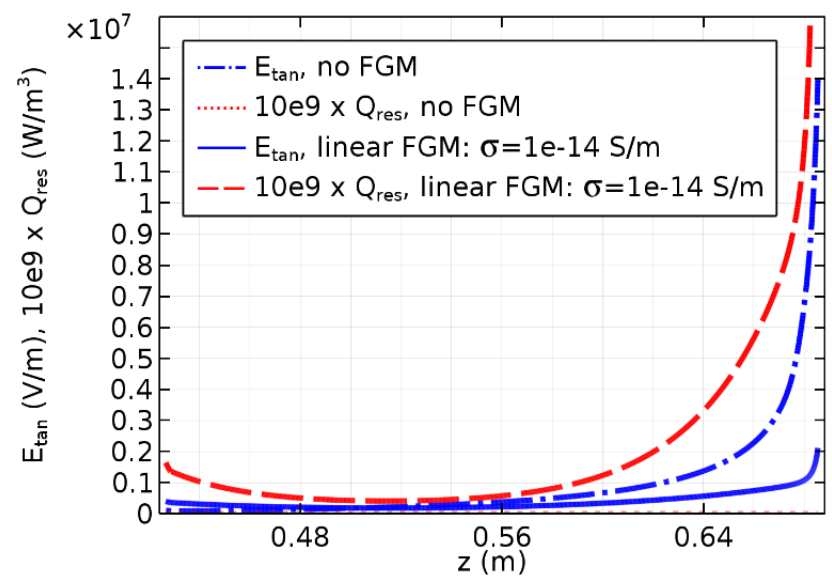

Fig. 3. Tangential electric field and resistive heating density along the outer surface of the FGM tube, without FGM and with linear FGM. 


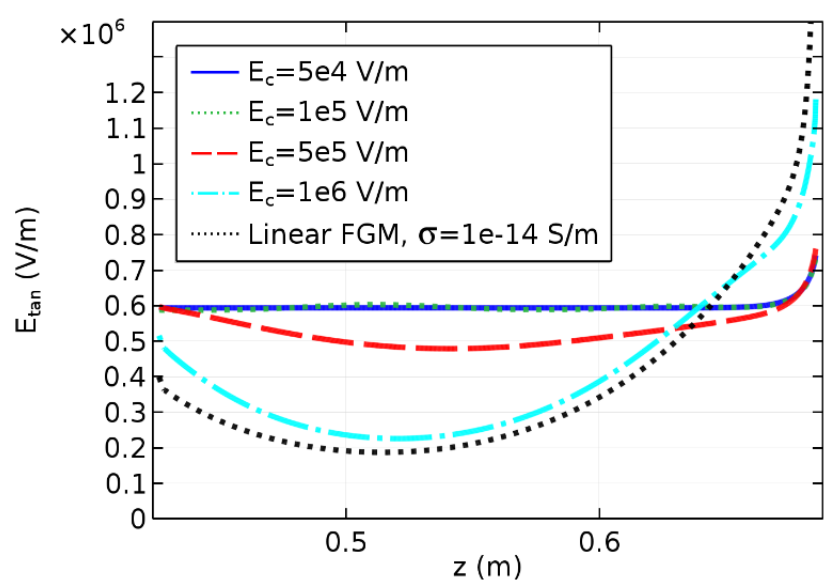

Fig. 4. Tangential electric field along the outer surface of the FGM tube. Comparison for different values of $E_{c}$. Saturation not considered.

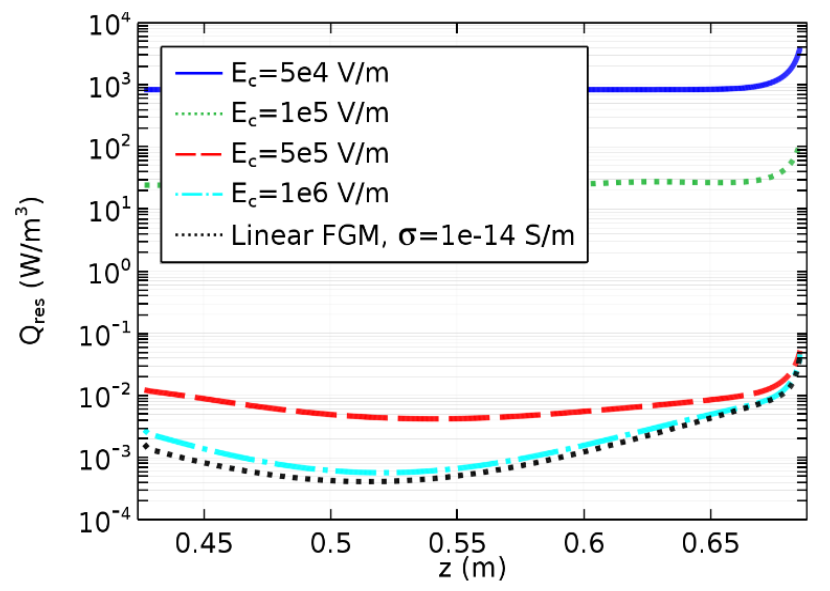

Fig. 5. Resistive heating density along the outer surface of the FGM tube. Comparison for different values of $E_{c}$. Saturation not considered.

FGM. It is seen that the maximum field stress is lowered from $1.4 \times 10^{7} \mathrm{~V} / \mathrm{m}$ down to $0.2 \times 10^{7} \mathrm{~V} / \mathrm{m}$. However, at the same time the heating density is increased from zero to a non-zero value. Increasing the conductivity further will not improve the field grading. The heat generation will, on the other hand, increase with higher conductivity.

Next we consider a nonlinear FGM, the conductivity of which is described by (1). No saturation is therefore included at this point. In Fig. 4, the tangential electric field distribution is plotted for different values of the threshold $E_{c}$, where the nonlinear increase of the conductivity starts. It is seen that for $E_{c} \leq 5 \times 10^{5} \mathrm{~V} / \mathrm{m}$ the maximum stress is constant while for $E_{c}>$ $5 \times 10^{5} \mathrm{~V} / \mathrm{m}$ it increases rapidly with $E_{c}$. Looking at Fig. 5, we also observe that the heating density starts to rapidly increase as $E_{c}$ is lowered below $5 \times 10^{5} \mathrm{~V} / \mathrm{m}$. For the presently studied application and applied voltage the combination of material parameters $\sigma_{0}=1 \times 10^{-14} \mathrm{~S} / \mathrm{m}$ and $E_{c} \approx 5 \times 10^{5} \mathrm{~V} / \mathrm{m}$ therefore represents an optimized FGM (assuming $\alpha=5$ and no saturation). It is interesting to note that although the field stress $E_{c}$ varies only by an order magnitude, the heating density and total heat generated increase by several orders of magnitude. This is due to the change in conductivity close to one of the electrodes strongly influencing the total current and hence the heat generation in the entire FGM volume.

\section{FGM WITH SATURATION}

If the material is allowed to saturate at high fields, i.e. expression (1) for the DC conductivity is replaced by the more general (2), the effect of varying $E_{s}$ depends on what value $E_{c}$ takes. Clearly, there must be regions where $E$ is of the same order as, or higher than, $E_{s}$ in order for saturation to have influence on the field-grading efficiency. For the bushing model under present study, it turns out that when $E_{c}$ is significantly higher than $1 \times 10^{5} \mathrm{~V} / \mathrm{m}$ saturation is insignificant. The results for $E_{c}=1 \times 10^{5} \mathrm{~V} / \mathrm{m}$ can be seen in Fig. 6 for the tangential electric field (close-up of the region close to the upper electrode) and the resistive heating density is presented in Fig. 7. If the saturation knee point $E_{s}$ is sufficiently close to $E_{c}$, a significant degradation of the field grading is observed. Fig. 7 demonstrates that even with a relatively small change in $E$, very close to the upper electrode, a large variation in heating density results all along the FGM tube.

The critical parameters are the maxima of the field stress and the heating density, which in our model always can be

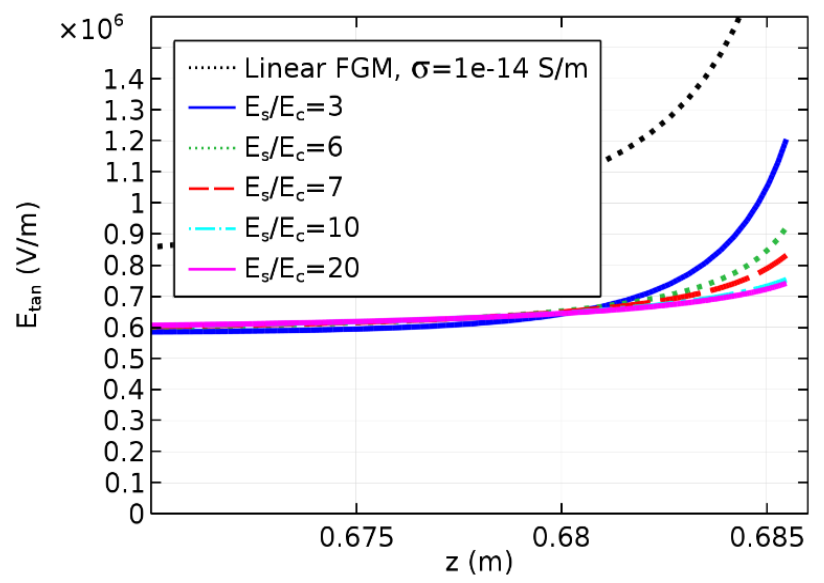

Fig. 6. Tangential electric field along the outer surface of the FGM tube close to the upper electrode. Comparison for different values of $E_{s}$ when $E_{c}=$ $1 \times 10^{5} \mathrm{~V} / \mathrm{m}$

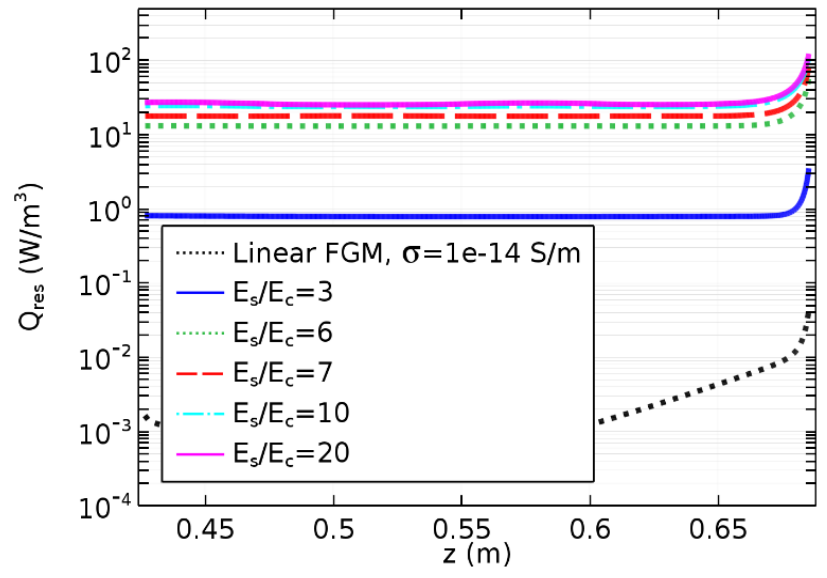

Fig. 7. Resistive heating density along the outer surface of the FGM tube. Comparison for different values of $E_{s}$ when $E_{c}=1 \times 10^{5} \mathrm{~V} / \mathrm{m}$. 


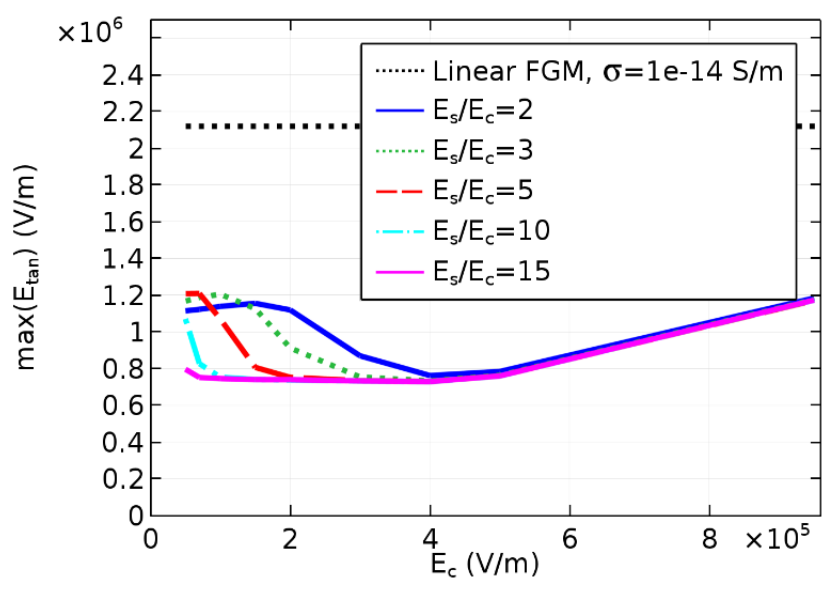

Fig. 8. Maximum tangential electric field along the outer surface of the FGM tube. Comparison for different values of $E_{s}$ and $E_{c}$.

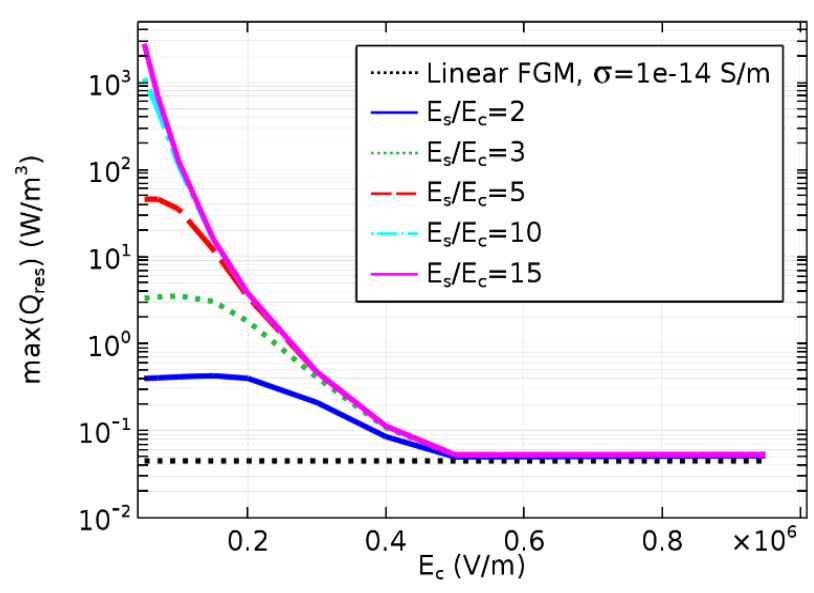

Fig. 9. Maximum resistive heating density along the outer surface of the FGM tube. Comparison for different values of $E_{s}$ and $E_{c}$.

found close to the upper electrode, which is at the same potential as the inner, high-voltage conductor. It is therefore sufficient to study how these maxima depend on the FGM parameters $E_{c}$ and $E_{s}$. A set of simulations were performed, where these two parameters were varied independently. The resulting behavior of $\max \left(E_{\text {tan }}\right)$ and $\max \left(Q_{\text {res }}\right)$ are displayed in Fig. 8 and Fig. 9, respectively. From Fig. 8, one can infer that with decreasing value of $E_{c}$, while keeping the ratio $E_{s} / E_{c}$ constant, the field-stress reduction becomes more and more efficient until the most stressed FGM parts start to saturate. Then the maximum field stress starts to increase again. In our particular example, choosing a material with $E_{c} \approx 4 \times 10^{5} \mathrm{~V} / \mathrm{m}$ seems to provide an optimal and robust solution. Above that value the stress is higher since less FGM volume is pushed into the nonlinear regime, while for lower values saturation may come into play and weaken the field-grading effect.

Although Fig. 8, indicates a general, unfavorable effect of saturation on field-stress reduction, Fig. 9 demonstrates that it lowers the heating density. The latter effect is, however, rather small at the optimal point $E_{c} \approx 4 \times 10^{5} \mathrm{~V} / \mathrm{m}$. Finally, the total heat generated in the FGM volume behaves similarly to the maximum local heating density shown in Fig. 9.

\section{CONCLUSIONS}

A rotationally symmetric computer model of a high-voltage DC bushing has been used to study the effect of the high-field saturation of an FGM on its electric-field grading ability and on resistive heating. In the case under study, an optimal value of the nonlinearity threshold $E_{c}$ exists at which both the electric stress is minimized and the FGM remains insensitive to possible saturation effects. Finally, it should be stressed that due to the nonlinear nature of the problem, the validity of this conclusion may depend on parameters such as geometry, applied voltage, low-field conductivity $\sigma_{0}$, and nonlinearity coefficient $\alpha$. We therefore recommend numerical simulations for estimation of the electric stress distribution and heating density in every single application.

\section{REFERENCES}

[1] Christen, T., Donzel, L., and Greuter, F., "Nonlinear resistive electric field grading, Part 1: Theory and simulation", IEEE Electr. Insul. Mag., vol. 26, no. 6, pp. 47-59, Nov. 2010.

[2] Christen, T., Donzel, L., and Greuter, F., "Nonlinear resistive electric field grading, Part 2: Materials and applications", IEEE Electr. Insul. Mag., vol. 27, no. 2, pp. 18-29, Mar. 2011.

[3] Wang, Z., Nelson, J.K., Hillborg, H., Zhao, S., and Schadler, L.S., "Nonlinear Conductivity and Dielectric Response of Graphene Oxide Filled Silicone Rubber Nanocomposites", Proceedings of the 2012 Conference on Electrical Insulation and Dielectric Phenomena (CEIDP 2012), pp. 40-43, Montreal, Canada, 2012.

[4] Sonerud, B. et al., "Impulse and step voltage measurements on materials with non-linear V-I characteristic", Proceedings of the 2012 Conference on Electrical Insulation and Dielectric Phenomena (CEIDP 2012), pp. 207-210, Montreal, Canada, 2012.

[5] Eriksson, G., Greijer, H., Pradhan, M., and Unge, M., "Stress Dependent Conductivity of Field Grading Materials under Time Varying Electrical Fields", Proceedings of the 2012 Conference on Electrical Insulation and Dielectric Phenomena (CEIDP 2012), pp. 165-169, Montreal, Canada, 2012.

[6] Eriksson, G., "Coupled Electric/Thermal/Fluid Analysis of High Voltage Bushing", Proceedings of COMSOL Conference 2012, Milan, Oct. 10-12, 2012.

[7] COMSOL software, htpp://www.comsol.com. 